\title{
The chemopreventive effect of Ginkgo biloba extract 761 against cisplatin ototoxicity: a pilot study
}

\author{
Mirela Alves Dias ${ }^{1,2}$ \\ André Luiz Lopes Sampaio ${ }^{1}$ \\ Alessandra Ramos Venosa ${ }^{1}$ \\ Elienai de Alencar Meneses ${ }^{2}$ \\ Carlos Augusto Costa Pires Oliveira
}

\begin{abstract}
Objective: The purpose of this study was to evaluate, by using distortion product otoacoustic emission test, the action of Ginkgo biloba extract 761, which has a known antioxidant property, as a possible otoprotective against cisplatin induced hearing loss. This prospective and double blinded study was conducted on individuals that were to begin cisplatin treatment in a tertiary university center. They were randomized and allocated in two groups: control group (CG) $(n=07)$ receiving placebo and cisplatin and study group (SG) $(n=08)$ receiving Ginkgo biloba extract 761 (240 mg/day) and cisplatin. Methods: This prospective study was conducted on individuals that were treated by cancer with cisplatin (CDDP) in a tertiary University center. Both groups were instructed to ingest the either placebo or Ginkgo biloba extract 761 before the first cisplatin dose. They were rated and followed up for approximately 90 days. The maximum cumulative cisplatin dosage was $300 \mathrm{mg} / \mathrm{m}^{2}$. The ototoxic effect was measured with distortion product otoacoustic emissions. Distortion product otoacoustic emissions were recorded before the first cisplatin cycle, 30, 60 and 90 days after the treatment. The average of the amplitude of the signals were calculated and used for comparisons between the groups. Results: Comparisons were made between baseline measurements and those recorded after maximum cumulative CDDP dose. The control group showed smaller distortion product otoacoustic emissions mean amplitudes and smaller signal/noise ratio than the study group $(p<0.05)$. Conclusion: Ginkgo biloba extract 761 probably has antioxidant properties, and might play otoprotective effect against cisplatin ototoxicity in these patients.
\end{abstract}

Keywords: cisplatin, ginkgo biloba, otoacoustic emissions, spontaneous

\footnotetext{
${ }^{1}$ University of Brasilia Medical School.

${ }^{2}$ Federal District Health Department.

Institution: University of Brasilia Medical School

Send correspondence to:

Mirela Alves Dias

SQNW 111 BL I ap 406, zip code - 70686745, Brasília DF, Brazil

Paper submitted to the RBCMS-SGP (Publishing Management System) on November 16, 2015;

and accepted on January 21, 2016. cod. 216
} 


\section{INTRODUCTION}

Cisplatin (cis-diamminedichloroplatinum) (CDDP) is a highly effective chemotherapeutic agent that is widely used to treat a variety of soft tissue neoplasms, including ovarian, testicular, cervical, head and neck, lung and bladder cancer. Serious side effects include nephrotoxicity, neurotoxicity and ototoxicity ${ }^{1,2}$.

In order to cure, the dose of CDDP has been increased in recent treatment protocols. Some audiometric studies have reported elevated hearing thresholds in $75-100 \%$ of patients treated with CDDP $^{3,4}$. This is particularly problematic in patients receiving CDDP with risk factors that increase the risk for ototoxicity that include: younger age, larger cumulative doses, pre-existing hearing loss, nephrologic disease and irradiation of the brain or skull base ${ }^{3,4}$.

The reported incidence of hearing loss associated with CDDP administration in adults ranges from $4 \%$ to $50 \%{ }^{3}$. However, if ultra-high frequency audiometry is used, the incidence of threshold changes may be as great as $100 \%^{1,5}$. The ototoxic effect in adults is characterized by irreversible, progressive, bilateral high-frequency sensorineural hearing loss associated with tinnitus ${ }^{5}$. The degree of hearing loss seems to be associated with cumulative dose, method of administration, previous cranial radiation, and age of the patient, with younger and older patients more commonly affected ${ }^{5}$.

The CDDP interacts with cochlear tissue and generates reactive oxygen species (ROS) such as superoxide anion. Cochlear tissues from animals receiving ototoxic doses of cisplatin were depleted of glutathione and antioxidant enzymes ${ }^{1}$. Inhibition of antioxidant enzyme activity by cisplatin can allow ROS, such as superoxide and hydrogen peroxide, and toxic lipid peroxides to increase within the cochlea. This can lead to calcium influx within cochlear cells, leading to apoptosis ${ }^{1}$.

Ultrastructural examination of human temporal bones has shown that the primary site of the CDDP ototoxicity is the outer hair cells (OHCs) of the basal and middle turns of the cochlea. The supporting cells and the stria vascularis are generally preserved ${ }^{5}$. Changes in spiral ganglion cells have also been reported in the basal turn, where OHCs damage is most extensive.

Although the treatment with this drug is often effective, serious side-effects such as nausea, nephrotoxicity, neurotoxicity and ototoxicity occur often. These side effects interfere with the treatment and often force the reduction of the dose, frequency and duration of the CDDP therapy ${ }^{2}$.

Considerable data indicate that distortion product otoacoustic emissions (DPOAEs) can provide information regarding the functional status of $\mathrm{OHCs}^{6,7}$. With evidence that CDDP preferentially affects OHCs function, it is possible to believe that DPOAEs would change with CDDP treatment ${ }^{8-11}$. Because of that, we used DPOAEs for monitoring CDDP ototoxicity in this clinical trial.

A number of chemoprotective agents have been evaluated in experimental and/or clinical studies to ameliorate CDDP induced toxicity.

Gingko biloba extract 761 (GBE 761) is a known extract with antioxidant effects that might prevent the ototoxic effects of cisplatin. GBE 761 was found to eliminate free oxygen radicals and therefore it might block oxidative stress and the hair cells apoptosis ${ }^{12,13}$. To our knowledge, by now, there are no studies in humans dealing with the antioxidant properties of GBE 761 in preventing $\mathrm{OHCs}$ injuries in patients undergoing to CDDP therapy.

We performed the present study in attempting to clarify the role of GBE 761 in the preventing OHCs damage caused by CDDP therapy in patients treated for cancer by means of DPOAEs.

\section{PATIENTS AND METHODS}

This study was submitted and approved by the ethic committee on human beings research at the University of Brasília - Brazil and the patients signed an informed authorization term in order to be included in the research protocol.

We performed a clinical trial - randomized, prospective double blind. The general selection criteria were: Age 18 years and older, no previous history of middle and inner ear diseases, no previous treatment with CDDP, normal audiometric and impedance audiometric tests as well as normal DPOAEs in the range of frequencies studied.

Patients were sent to oncologic treatment from the regional public hospitals and were approached to follow this protocol as they signed the consent permition. Then fifteen consecutive patients were enrolled in this clinical trial. These patients were selected for CDDP treatment based on their oncologic treatment indication at the oncologic clinic at the University of Brasilia Hospital and Hospital de Base in Brasília, Brazil. The patients were randomly selected into two groups.

The study group (SG) was composed by 8 subjects undergoing to CDDP treatment for cancer and GBE 761 (120 mg twice a day) and the control group (CG) was formed by 7 individuals that received CDDP also for cancer treatment and placebo (twice a day). Although a dose-response relationship is not established, 240 mg day GBE seems to show a higher rate of treatment response than other doses of $\mathrm{GBE}^{14}$. Pharmacological experiments conclude that 50 or $100 \mathrm{mg} / \mathrm{kg} / \mathrm{p} .0 /$ day is usually active. In humans, 120 or $240 \mathrm{mg} /$ day seems to be active in chronic treatment ${ }^{15}$. Futhermore, there is not 
linearity increase of the GBE 761 responses according to the increase of dosage. That is, doses higher than $240 \mathrm{mg}$ may not improve the effects of treatment ${ }^{14}$. The CG and SG subjects received either placebo that were pills manufactured by pharmaceutic laboratory of Hospital Regional da Asa Norte in Brasília and or GBE 761 is a standardized GB extract manufactured by pharmaceutical standards by Dr. Willmar Schwabe Company, Germany, that were donated to the researchers.

The placebo was very similar to the GBE 761 and so the researchers and the patients could not tell one from the other and did not know the pills allocated for each patient. The individuals were required to intake either placebo or GBE 761 two hours before the first CDDP dose and to keep the dosage during the whole CDDP treatment. GBE 761 can have effects within a short period of time (ie, within 1 to 3 hours from the intake) ${ }^{16}$. According to them, GBE 761 exhibited half lives ranging from 2 to 4 hours and activity levels with peak at 1.5 to 3 hours in animal and human models. The subjects were followed up weekly so the researcher could supervise the oral intake of either pills in the two groups.

Pure tone audiometry (PTA), impedance audiometry and DPOAEs tests were performed just previously to the beginning of CDDP treatment in SG and CG. PTA was recorded at the frequencies of $0.25,0.5,1,2,3,4$, 6 , and $8 \mathrm{KHz}$ in a cabin by using an AD-229E Clinical Audiometer - Interacoustics ${ }^{\circledR}$ and the standard TDH 39P ear phone. The AZ7. Imitanciometer from Interacoustics ${ }^{\circledR}$ was used for the imitanciometry.

PTA thresholds were considered normal if less than $25 \mathrm{~dB}$ as referred by Silman and Silverman in $1997^{17}$. Normal tympanogram type $A$ and presence of ipsi and contralateral stapedial reflex were the normal parameters for impedance audiometry.

DPOAEs were recorded by using a Scout biologic equipment from bio-logic systems ${ }^{\circledR}$ with the patient sitting in a silent room. We employed the $750-8000 \mathrm{~Hz}$ diagnostic protocol. F2 frequency ranged from 750 to $8000 \mathrm{~Hz}$ in $1 / 2$ octave steps, and the ratio (f2/f1) was equal to 1.22. The primary levels were set with $L 1=65$ $\mathrm{dBSPL}$ and $\mathrm{L} 2=55 \mathrm{dBSPL}$. A DPOAE was considered to be present if its level was $\geq 8 \mathrm{~dB}$ above that of the related noise floor. Gorga criteria were used for the result analysis of the amplitude of signal and signal/noise ratio $^{18}$. Cumulative distribution responses from normal and abnormal ears according to 90 and 10 percentiles were employed for the result analysis.

DPOAEs were recorded before the first CDDP cycle, 30,60 and 90 days after the treatment. The average of the amplitude of the signals were calculated and used for comparisons between the groups.

CDDP therapy followed protocols for treatment of different solid tumors and the cumulative dose ranged from 50 to $300 \mathrm{mg} / \mathrm{m}^{2}$. The patients were reallocated into 3 subgroups according to the cumulative doses: $40-150$ $\mathrm{mg} / \mathrm{m}^{2}, 150-225 \mathrm{mg} / \mathrm{m}^{2}, 225-300 / \mathrm{m}^{2}$ for the statistical analysis.

The ototoxic effect of CDDP is reported to be bilateral and symmetric. The right ears were grouped with left ears among the SG and CG.

The following analyses were performed in order to investigate the possible ameliorate CDDP induced toxicity:

The average of the amplitude of the signal and signal-noise $(\mathrm{S} / \mathrm{N})$ ratio per frequencies before the CDDP initial dose and after the cumulative dose.

The average of the amplitude of the signal and $\mathrm{S} / \mathrm{N}$ ratio per cumulative dose groups.

Analyse of amplitude of the signal and $\mathrm{S} / \mathrm{N}$ ratio according to the normality parameters for percentiles 90 and 10.

All analyses were performed for the SG and CG. The results of the groups were compared by U-ManWhitney, Kruskal Wallis and $t$ Student test by employing. Minitab 13 and SPSS 11 softwares. P value was considered relevant if $P$ was $<0.05$.

U-Man Whitney test was employed for comparison of the average of amplitude of the signal and $\mathrm{S} / \mathrm{N}$ ratio between SG and CG. Kruskal Wallis test was used to investigate the differences between the groups of cumulative doses of CDDP. $T$ student test was used for the study of the normality parameters according to percentiles 10 and 90 in the SG and CG.

\section{RESULTS}

Among 15 patients followed up, the age ranged from 24 to 69 years old. There was no statistically significant difference $(p>0.05)$ between the groups according to the age and the analysis of the amplitude of the signal in the pre-treatment evaluation in all frequencies. However, there was a statistical significant difference between the $C G$ and $S G$ after CDDP treatment for the frequency of $8 \mathrm{KHz}(p=0.03)$ (Figure 1).

There was no statistical significant difference among the subgroups of cumulative doses of CDDP in the $C G$ and in the $S G$ in the amplitude of the DPOAEs in the range of frequencies studied $(p>0.05)$ (Figures $2 \mathrm{~A} ; 2 \mathrm{~B})$.

When we compared the initial amplitude of the signal of DPOEs, regarding the 90 and 10 percentiles, before the total cumulative dose in the CG (Figure $3 \mathrm{~A}$ ) with the post cumulative dose we found a statistical significant difference for $8 \mathrm{KHz}$ frequency $(p=0.04, t$-Student Test) (Figure $3 \mathrm{C}$ ). There was no significant difference for the other frequencies studied $(p>0.05)$. Study group showed no relevant difference in the range of frequencies evaluated according to 90 and 10 percentiles when we 


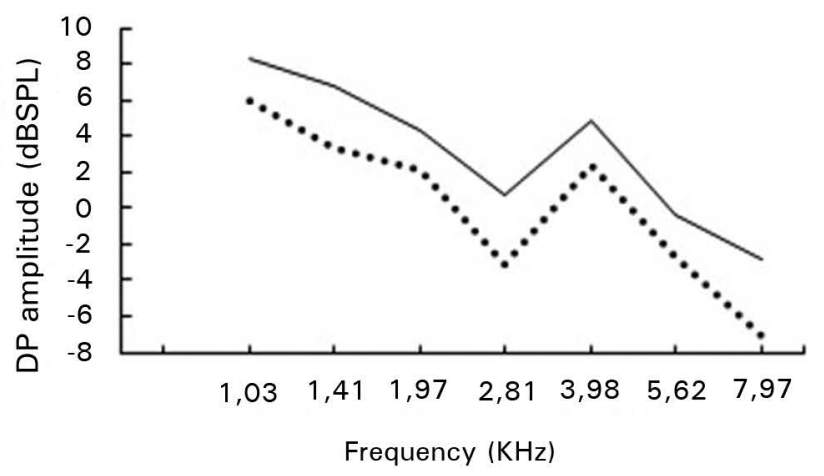

A $\quad$ Control Group $\quad \ldots . .$. Study Group

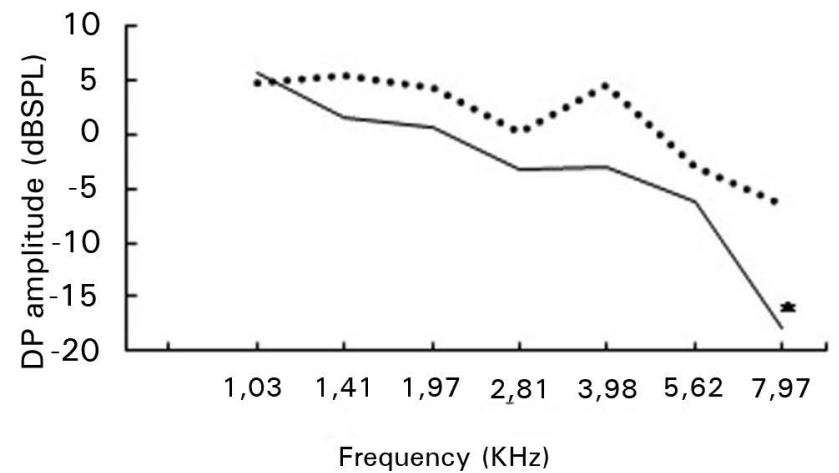

B
Study Group

Figure 1. A: Graph showing the comparison of mean DPOAEs before cisplatin injection between study and control groups in the range of frequencies studied ( $p>0.05$, U-ManWhitney Test). B: Comparison of mean DPOAEs after maximum cumulative dose of cisplatin $\left(225-300 \mathrm{mg} / \mathrm{m}^{2}\right) \mathrm{between}$ the study and control groups in the range of frequencies studied ( ${ }^{*} p=0.03$, U-ManWhitney Test).

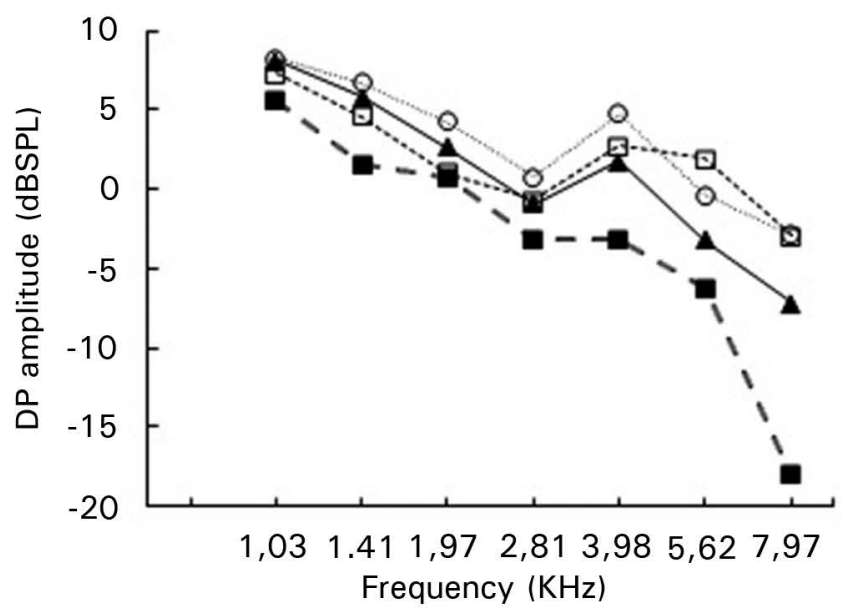

A

$$
\begin{array}{ll}
\longrightarrow-40 \dashv 150 \\
\cdots-150 \dashv 225 & - \pm-225 \dashv 300
\end{array}
$$
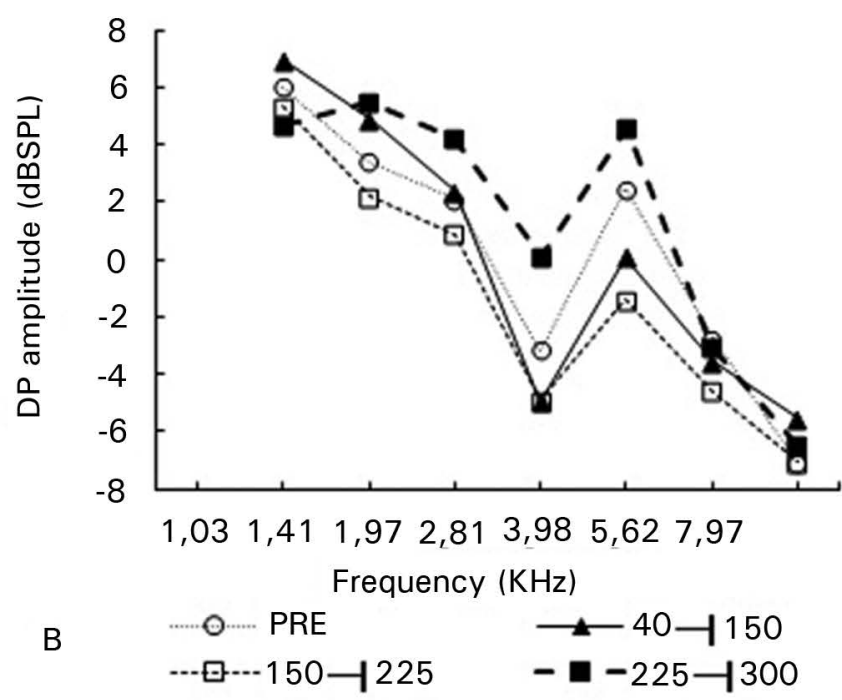

Figure 2. A: Graph showing the mean DPOAE changes in control group after cisplatin injection among different cumulative doses in the range of frequencies studied ( ${ }^{*} p>0.05$, KruskalWallis Test). B: Graph showing the mean DPOAE changes in study group after cisplatin injection among different cumulative doses in the range of frequencies studied ( $p>0.05$, KruskalWallis Test).

compared pre and post total CDDP cumulative dose ( $p$ $>0.05, t$-Student Test) (Figures 3B; 3D).

There was no statistically significant difference $(p>0.05)$ between the $C G$ and SG according to the analysis of the $\mathrm{S} / \mathrm{N}$ ratio in the pre-treatment evaluation in all frequencies.

Control group showed a statistical significant decrease in the $\mathrm{S} / \mathrm{N}$ ratio in the $8 \mathrm{KHz}$ frequency in the maximum CDDP cumulative dose $\left(225-300 \mathrm{mg} / \mathrm{m}^{2}\right)(p=$ $0.04)$ (Figure 4). Study group showed no difference in the $\mathrm{S} / \mathrm{N}$ ratio in the range of frequencies evaluated among different CDDP cumulative doses $(p>0.05)$.
When we compared CG with the SG for the post total CDDP cumulative dose, among all subgroups of doses, we found a statistical significant difference between the groups in the frequency of $8 \mathrm{KHz}(p=0.03)$ (Figure 5A; 5B). CG showed lower $\mathrm{S} / \mathrm{N}$ ratio compared to SG post CDDP treatment.

No side effects were observed from GBE761 or placebo. In patients of SG, GBE 761 did not interfere with the antitumor activity of CDDP as registred by the patients records. This might be in agreement to the fact that GBE 761 has been shown to possess proapoptotic activity against malignant neoplasms ${ }^{19}$. 

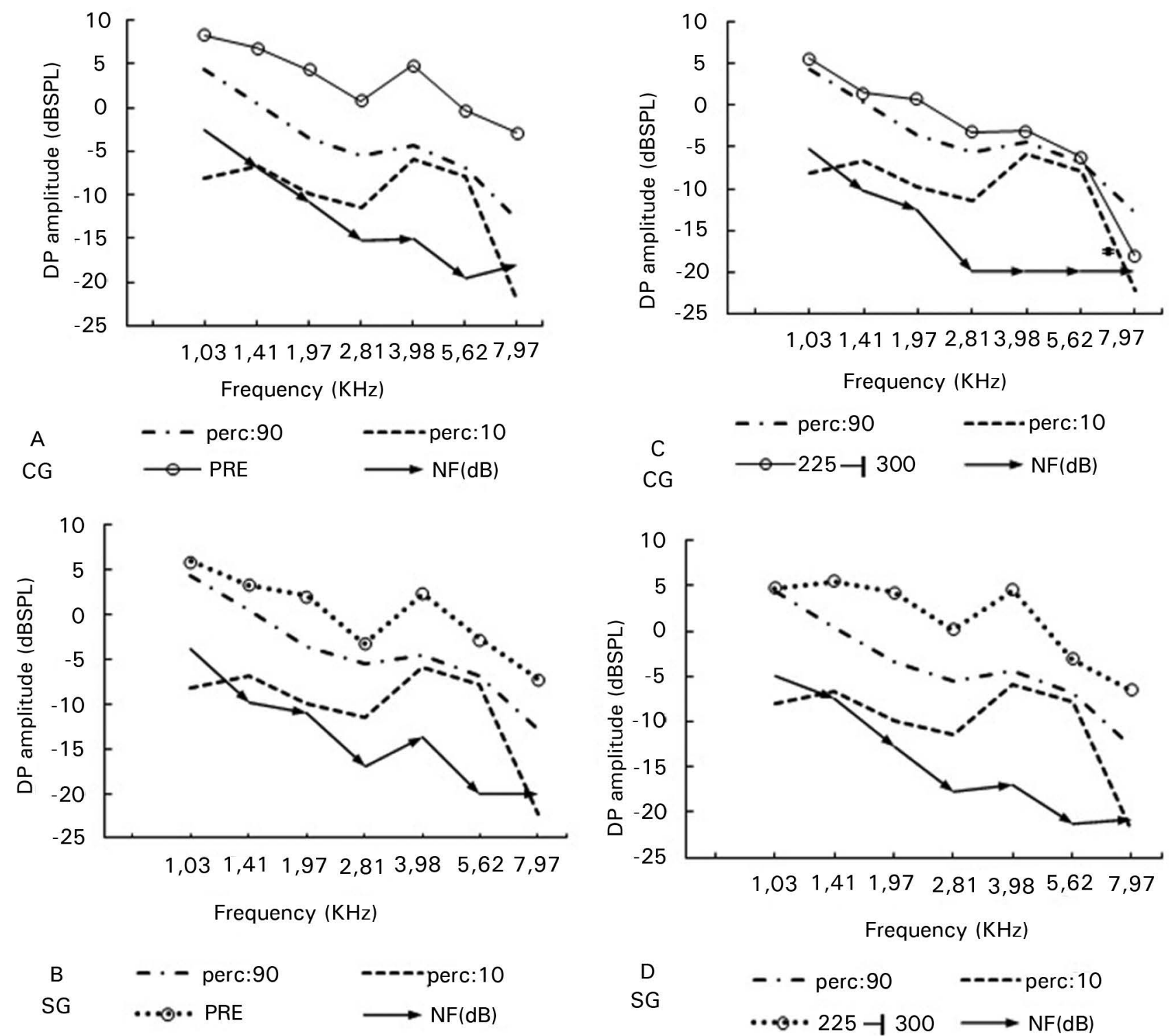

Figure 3. A: Graph showing the mean DPOAE in control group (CG) before cisplatin injection (pre/solid line with open circles) according to percentiles $90^{\text {th }}$ and $10^{\text {th }}$. Noise amplitude (NF) is shown as solid line with arrows. B: The mean DPOAE in study group (SG) before cisplatin injection according to percentiles 90th and $10^{\text {th }}$.(C) The mean DPOAE changes in control group(CG) after maximum dose $\left(225-300 \mathrm{mg} / \mathrm{m}^{2}\right)$ according to percentiles $90^{\text {th }}$ and $10^{\text {th }}$ in the range of frequencies studied $\left({ }^{*} p=0.04, T\right.$-Student Test).(D) The mean DPOAE in study group (SG) after maximum dose $\left(225-300 \mathrm{mg} / \mathrm{m}^{2}\right)$ of cisplatin according percentiles $90^{\text {th }}$ and $10^{\text {th }}(p>0.05, T$-Student Test).

\section{DISCUSSION}

Cisplatin therapy is a well known treatment for cancer and the side effects include: gastrointestinal toxicity, neurotoxicity, nephrotoxicity and ototoxicity. The ototoxicity and neurotoxicity are still a dose-limited problem for the patients.

It was not uncommon to find loss in higher frequencies; nonetheless, continuous administration of this agent increases loss, and other frequencies are altered. It should be noted that eventually speech frequencies will be affected ${ }^{5,18}$.
In the present study we employed DPOAE test that is recognized as a fast, non-invasive and reliable method to follow early cochlear changes. In our study CG showed OHCs abnormalities in $8 \mathrm{KHz}$ at the maximum CDDP cumulative dose $\left(225-300 \mathrm{mg} / \mathrm{m}^{2}\right)$. This is in accordance to the literature ${ }^{5,18}$. SG had no significant changes in DPOAE at the same CDDP cumulative dose.

The frequency of $8 \mathrm{KHz}$ was previously reported to be the first area to change in DPOAEs ${ }^{5}$. In our sample it was the only one frequency to change. Our patients were followed up to 90 days and it is possible that the 

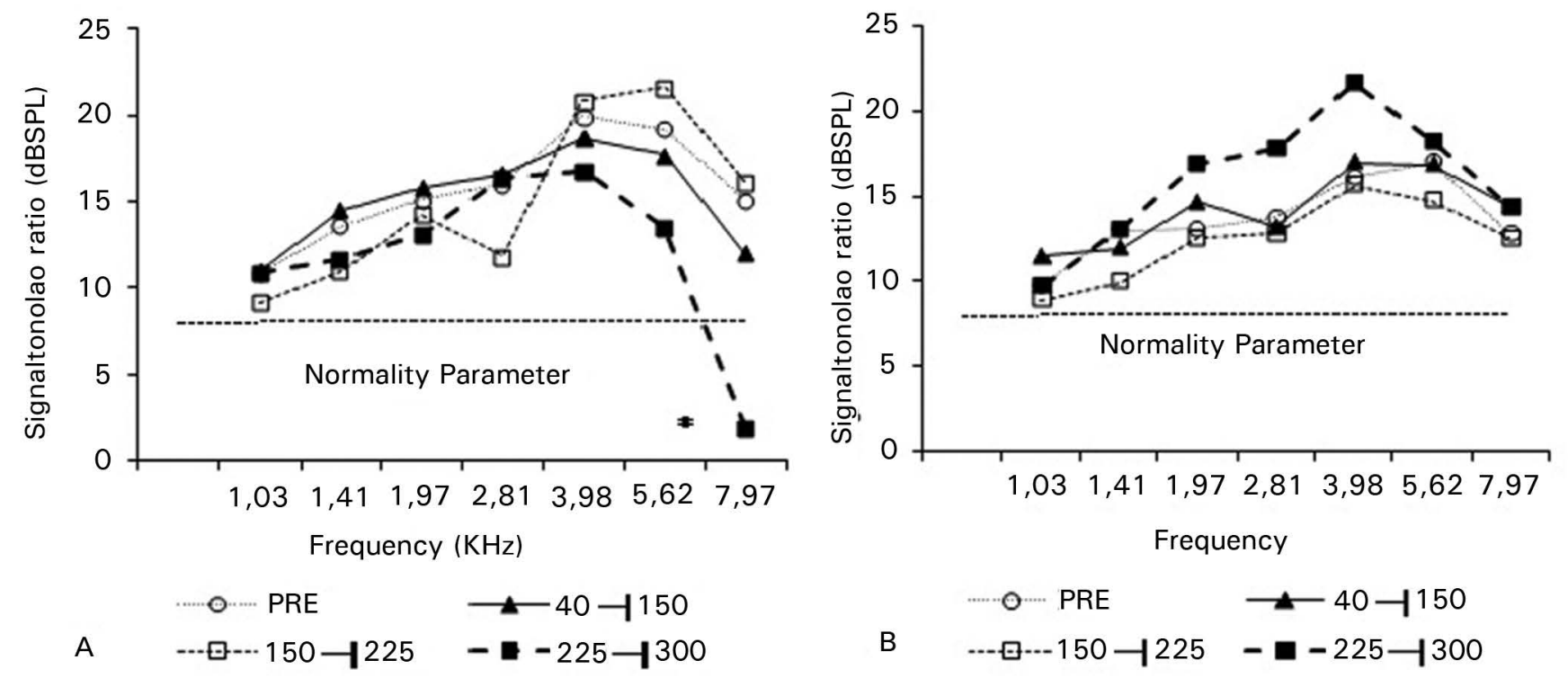

Figure 4. A: Graph showing mean signal/noise ratio changes in the control group among different cumulative cisplatin doses in the range of frequencies studied ( ${ }^{*} p=0.04, T$-Student Test.(B) The mean signal/noise ratio in the study group among different cumulative cisplatin doses in the range of frequencies studied $(p>0.05, T$-Student Test).

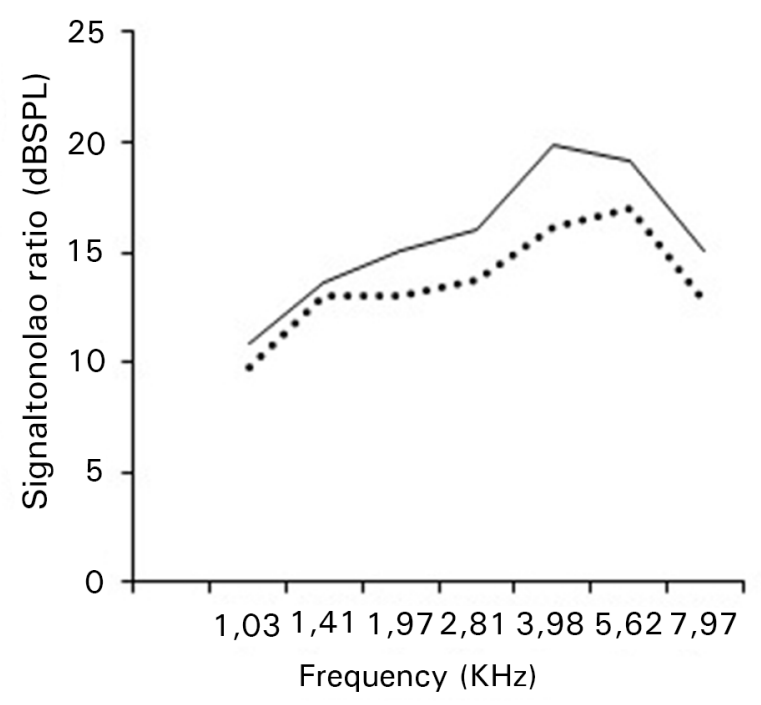

A Control Group $\quad \cdots . .$. Study Group

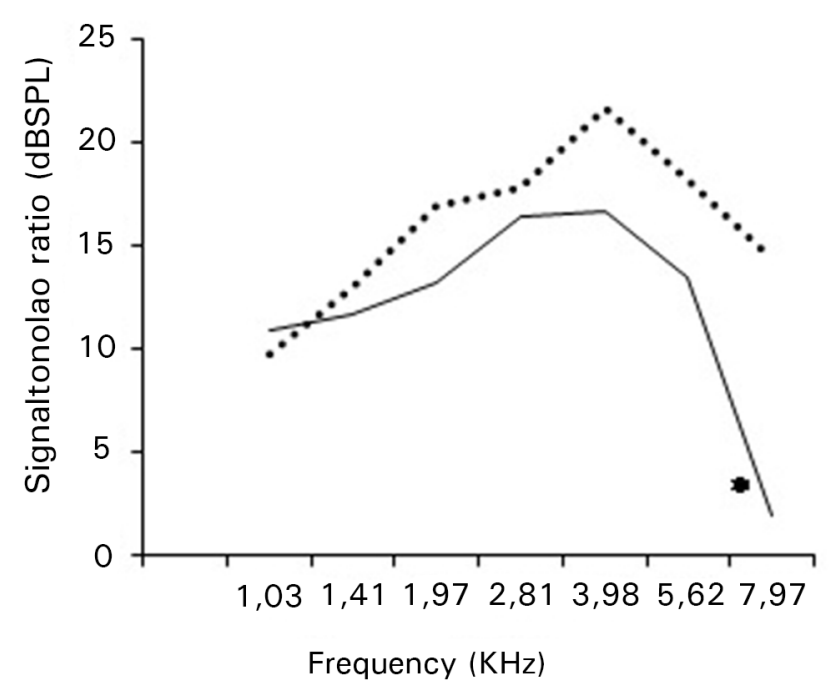

B Control Group $\quad$....... Study Group

Figure 5. A: Graph showing the comparison of mean signal/noise ratio before cisplatin injection between study and control groups in the range of frequencies studied ( $p>0.05$, U-ManWhitney Test). B: Graph showing the comparison of mean signal/noise ratio after maximum cumulative dose of cisplatin $\left(225-300 \mathrm{mg} / \mathrm{m}^{2}\right)$ between study and control groups among all subgroups of cumulative doses in the range of frequencies studied $\left({ }^{\star} p=0.03\right.$, U-ManWhitney Test).

deposit effect of CDDP in the body could not be evaluated among our patients because of the short term follow up period. However, it was sufficient to show some ototoxic effect since it usually appears during the first days of the drug administration. The sustained otoprotective effect of GBE 761 could not be either assessed in this study.
Clinical studies showed that ototoxic effect of CDDP depended on total dose administered in all sessions. For this reason we separated the ears in different groups of cumulative doses. Hearing loss in high frequencies was generally present at a dose of $200 \mathrm{mg} / \mathrm{m}^{2}$ or higher ${ }^{5,20}$. It has been observed that hearing loss may occur even after several 
days after CDDP administration ${ }^{21,22}$. In this short term clinical follow up we detected a statistical significant decrease in the amplitude of the signal and $\mathrm{S} / \mathrm{N}$ ratio in the isolated frequency of $8 \mathrm{KHz}$ in the $\mathrm{CG}$ after the total cumulative dose of CDDP. It is possible if we had tested the patients longer after, other frequencies might become affected.

For these reasons it is rational to search for new protective drugs that might ameliorate CDDP therapy toxic effects in order to become it well tolerated to the patients suffering from cancer ${ }^{23}$. In our study we tested the protective effect of GBE 761 in the OHCs of human beings by means of DPOAE record. Animal studies attempting to investigate the protective effect of GBE 761 against CDDP therapy used doses of CDDP that sometimes could not be compared to doses used clinically ${ }^{12,13}$. The route of administration of CDDP (e.g subcutaneous, intraperitoneal and intramuscular) differs markedly from the humans. The extrapolation of these findings to humans has been difficult. Therefore we decided to perform this clinical trial in order to test the otoprotective effect of GBE 761 against CDDP toxic effects in the humans OHCs.

The most part of the clinical studies are concentrated in the early diagnostic methods to detect ototoxicity in subjects using CDDP. Just a few studies were designed to evaluate the chemoprotective drugs against CDDP ototoxicity.

To our knowledge, this is the first study with patients undergoing to CDDP anti-cancer therapy and GBE 761. The ototoxicity is reported to be due to superoxide products formation that leads to the OHCs apoptosis. GBE 761 is a dietary supplement derived from the deciduous tree, Ginkgo biloba, considered to be the world's oldest living tree species, which contains unique chemical substances unknown in other living things ${ }^{13}$.

We used the original GBE 761 from Dr. Willmar Schwabe Pharmaceuticals ${ }^{\circledR}$ in Germany in the patients of $S G$ in our study.

One of the proposed mechanisms for its beneficial pharmacologic effect is its potent antioxidant action ${ }^{24,25}$. Studies have shown the in vitro and in vivo protective effects of GBE 761 against oxidative stress ${ }^{25,26}$, and these are closely related to its ability to scavenge free radicals, such as superoxide anion, hydroxyl, and peroxyl radicals $^{27,28}$. Furthermore, GBE 761 also inhibits lipid peroxidation reactions and enhances antioxidant enzyme activities ${ }^{12}$. The free radical scavenging activity of GBE 761 is comparable to that of other known antioxidants such as vitamins $A$ and $C$, as well as the tripeptide $\mathrm{GSH}^{29}$. GBE 761 also can scavenge nitric oxide and inhibit its production by nitric oxide synthase (NOS) ${ }^{30,31}$. In addition, GBE 761 has anti-inflammatory and antiapoptotic properties $^{32}$. The purpose of the present study was to examine the efficacy of the standardized GBE 761 in protecting against CDDP-induced ototoxicity in humans by means of DPOAE test.
The maximum CDDP cumulative doses (225-300 $\mathrm{mg} / \mathrm{m}^{2}$ ) in our patients showed toxic effects in the inner ear with significant decrease in the amplitude of the signal in $8 \mathrm{KHz}$ frequency in CG. In this study we attempted to investigate whether GBE 761 had otoprotective effect against CDDP ototoxic effect in the OHCs. Our SG received GBE $761120 \mathrm{mg}$ twice a day. There was no significant change in DPOAE in the SG.

These findings were supported by $\mathrm{OHC}$ preservation by GBE 761 in animals models demonstrated by scanning electron microscopy as compared with the marked $\mathrm{OHC}$ loss observed in animals receiving CDDP and no protection drug $^{12,13}$. Our data offer evidence supporting the potential benefits of the antioxidant GBE 761 in CDDP chemotherapy in the preservation of hearing in humans for the first time.

Our findings suggest that GBE 761 could protect against CDDP induced ototoxicity in this preliminary clinical trial and are consistent with a Japanese study which showed that a standardized GBE 761 administered 90 minutes before administration of CDDP could ameliorate drug-induced auditory threshold shifts and could protect renal function without any attenuation of the anticancer effect of CDDP in Fisher rats ${ }^{33}$.

The combined activity of all the EGB 761 components is required for optimal therapeutic action ${ }^{32}$. Further studies with long term follow up including more patients should be addressed in order to clarify the long term effects of GBE 761 on hearing preservation and antitumor properties.

By the other hand, CDDP ototoxicity affects different individuals in a widely variable manner. These variations are likely to be explained by genetic differences among those affected. It would be highly advantageous to identify genetic variants that predispose to CDDP ototoxicity in order to minimize the risk to susceptible subgroups. The most important limitation of our study and the data analysis was the size of our sample. First, the study was conducted using a small sample (30 ears), as are most ototoxicity studies and many other biological datasets. Analysis of performance of drugs in small samples might be impacted by the idiosyncratic nature of the sample and there is always the risk of overfitting the data making the results less generalizable.

Other studies allocating more patients should be addressed in order to minimize the bias of the individual predisposition to the toxic effects of CDDP in the auditory system and chemoprotective role of EGB 761 .

CDDP induced hearing loss might continue to develop days after the interruption of CDDP administration. We did not evaluate the DPOAE after the $90^{\text {th }}$ day. It is possible the toxic effects of CDDP on OHCs keep developing. GBE 761 might be effective in ameliorating the long term ototoxic action of CDDP. We strongly recommend long term evaluation of the hearing in the patients undergoing to CDDP antineoplastic therapy as well as GBE 761 otoprotective effects. 


\section{CONCLUSION}

GBE 761 at $240 \mathrm{mg}$ a day showed some otoprotection effect against CDDP induced OHCs damage evaluated by DPOAE. Further studies with more patients and long term follow up may reveal optimal dosing, administration via and potential clinical usefulness of GBE 761 as a chemoprotective agent against CDDP ototoxicity.

\section{REFERENCES}

1. Sie KC, Norton SJ. Changes in otoacoustic emissions and auditory brain stem response after cis-platinum exposure in gerbils. Otolaryngol Head Neck Surg. 1997;116(6 Pt 1):585-92.

2. van den Berg JH, Beijnen JH, Balm AJ, Schellens JH. Future opportunities in preventing cisplatin induced ototoxicity. Cancer Treat Rev. 2006;32(5):390-7. DOI: http://dx.doi.org/10.1016/j.ctrv.2006.04.011

3. Rybak LP, Ramkumar V. Ototoxicity. Kidney Int. 2007;72(8):931-5. PMID: 17653135 DOI: http://dx.doi.org/10.1038/sj.ki.5002434

4. Ress BD, Sridhar KS, Balkany TJ, Waxman GM, Stagner BB, Lonsbury-Martin BL. Effects of cis-platinum chemotherapy on otoacoustic emissions: the development of an objective screening protocol. Third place--Resident Clinical Science Award 1998. Otolaryngol Head Neck Surg. 1999;121(6):693-701. PMID: 10580222 DOI: http://dx.doi.org/10.1053/hn.1999.v121.a101567

5. Stavroulaki P, Apostolopoulos N, Segas J, Tsakanikos M, Adamopoulos G. Evoked otoacoustic emissions--an approach for monitoring cisplatin induced ototoxicity in children. Int $\mathrm{J}$ Pediatr Otorhinolaryngol. 2001;59(1):47-57. PMID: 11376818 DOI: http:// dx.doi.org/10.1016/S0165-5876(01)00455-4

6. Lonsbury-Martin BL, Whitehead ML, Martin GK. Clinical applications of otoacoustic emissions. J Speech Hear Res. 1991;34(5):964-81. PMID: 1749251 DOI: http://dx.doi.org/10.1044/jshr.3405.964

7. Biro K, Noszek L, Prekopp P, Nagyiványi K, Géczi L, Gaudi I, et al. Characteristics and risk factors of cisplatin-induced ototoxicity in testicular cancer patients detected by distortion product otoacoustic emission. Oncology. 2006;70(3):177-84. PMID: 16757924 DOI: http://dx.doi.org/10.1159/000093776

8. Stadnicki SW, Fleischman RW, Schaeppi U, Merriam P. Cis-dichlorodiammineplatinum (II) (NSC-119875): hearing loss and other toxic effects in rhesus monkeys. Cancer Chemother Rep. 1975;59(3):467-80. PMID: 812603

9. Laurell G, Engström B, Hirsch A, Bagger-Sjöbäck D. Ototoxicity of cisplatin. Int J Androl. 1987;10(1):359-62. DOI: http://dx.doi. org/10.1111/j.1365-2605.1987.tb00203.x

10. Knight KR, Kraemer DF, Winter C, Neuwelt EA. Early changes in auditory function as a result of platinum chemotherapy: use of extended high-frequency audiometry and evoked distortion product otoacoustic emissions. J Clin Oncol. 2007;25(10):1190-5. DOI: http://dx.doi.org/10.1200/JCO.2006.07.9723

11. Le Bars PL, Kastelan J. Efficacy and safety of a Ginkgo biloba extract. Public Health Nutr. 2000;3(4A):495-9. DOI: http://dx.doi. org/10.1017/S1368980000000574

12. Bridi R, Crossetti FP, Steffen VM, Henriques AT. The antioxidant activity of standardized extract of Ginkgo biloba (EGb 761) in rats. Phytother Res. 2001;15(5):449-51. DOI: http://dx.doi.org/10.1002/ptr.814

13. Huang $X$, Whitworth CA, Rybak LP. Ginkgo biloba extract (EGb 761) protects against cisplatin-induced ototoxicity in rats. Otol Neurotol. 2007;28(6):828-33. DOI: http://dx.doi.org/10.1097/ MAO.0b013e3180430163

14. Le Bars PL, Kastelan J. Efficacy and safety of a Ginkgo biloba extract. Public Health Nutr. 2000;3(4A):495-9. DOI: http://dx.doi. org/10.1017/S1368980000000574

15. Christen Y, Maixent JM. What is Ginkgo biloba extract EGb 761? An overview--from molecular biology to clinical medicine. Cell Mol Biol (Noisy-le-grand). 2002;48(6):601-11.
16. Diamond BJ, Shiflett SC, Feiwel N, Matheis RJ, Noskin O, Richards JA, et al. Ginkgo biloba extract: mechanisms and clinical indications. Arch Phys Med Rehabil. 2000;81(5):668-78. PMID: 10807109 DOI: http://dx.doi.org/10.1016/S0003-9993(00)90052-2

17. Silman S, Silverman CA. Basic audiologic testing. In: Silman S, Silverman CA, eds. Auditory Diagnosis: principles and applications. San Diego: Singular Publishing Group; 1997. p.44-52.

18. Gorga MP, Neely ST, Ohlrich B, Hoover B, Redner J, Peters J. From laboratory to clinic: a large scale study of distortion product otoacoustic emissions in ears with normal hearing and ears with hearing loss. Ear Hear. 1997;18(6):440-55. DOI: http://dx.doi. org/10.1097/00003446-199712000-00003

19. Kim KS, Rhee KH, Yoon JH, Lee JG, Lee JH, Yoo JB. Ginkgo biloba extract (EGb 761) induces apoptosis by the activation of caspase-3 in oral cavity cancer cells. Oral Oncol. 2005;41(4):383-9. PMID: 15792610 DOI: http://dx.doi.org/10.1016/j.oraloncology.2004.09.013

20. Rozencweig M, Von Hoff DD, Muggia FM. Investigational chemotherapeutic agents in head and neck cancer. Semin Oncol. 1977;4(4):425-9. PMID: 74096

21. Strauss M, Towfighi J, Lord S, Lipton A, Harvey HA, Brown B. Cis-platinum ototoxicity: clinical experience and temporal bone histopathology. Laryngoscope. 1983;93(12):1554-9. DOI: http:// dx.doi.org/10.1288/00005537-198312000-00007

22. Schweitzer VG. Ototoxicity of chemotherapeutic agents. Otolaryngol Clin North Am. 1993;26(5):759-89.

23. Laurell G, Beskow C, Frankendal B, Borg E. Cisplatin administration to gynecologic cancer patients. Long-term effects on hearing. Cancer. 1996;78(8):1798-804. PMID: 8859195 DOI: http://dx.doi.org/10.1002/ (SICI) 1097-0142(19961015)78:8<1798::AID-CNCR22>3.0.CO;2-S

24. Oyama Y, Chikahisa L, Ueha T, Kanemaru K, Noda K. Ginkgo biloba extract protects brain neurons against oxidative stress induced by hydrogen peroxide. Brain Res. 1996;712(2):349-52. PMID: 8814913 DOI: http://dx.doi.org/10.1016/0006-8993(95)01440-3

25. Rong Y, Geng Z, Lau BH. Ginkgo biloba attenuates oxidative stress in macrophages and endothelial cells. Free Radic Biol Med. 1996;20(1):121-7. DOl: http://dx.doi.org/10.1016/0891-5849(95)02016-0

26. Pincemail J, Dupuis M, Nasr C, Hans P, Haag-Berrurier M, Anton R, et al. Superoxide anion scavenging effect and superoxide dismutase activity of Ginkgo biloba extract. Experientia. 1989;45(8):708-12. PMID: 2547645 DOI: http://dx.doi.org/10.1007/BF01974564

27. Maitra I, Marcocci L, Droy-Lefaix MT, Packer L. Peroxyl radical scavenging activity of Ginkgo biloba extract EGb 761. Biochem Pharmacol. 1995;49(11):1649-55. PMID: 7786306 DOI: http://dx.doi. org/10.1016/0006-2952(95)00089-I

28. Yoshikawa T, Naito Y, Kondo M. Ginkgo biloba leaf extract: review of biological actions and clinical applications. Antioxid Redox Signal. 1999;1 (4):469-80. DOI: http://dx.doi.org/10.1089/ars.1999.1.4-469

29. Köse K, Doğan P. Lipoperoxidation induced by hydrogen peroxide in human erythrocyte membranes. 2. Comparison of the antioxidant effect of Ginkgo biloba extract (EGb 761) with those of water-soluble and lipid-soluble antioxidants. J Int Med Res. 1995;23(1):9-18.

30. Sharma HS, Drieu K, Alm P, Westman J. Role of nitric oxide in blood-brain barrier permeability, brain edema and cell damage following hyperthermic brain injury. An experimental study using EGB-761 and Gingkolide B pretreatment in the rat. Acta Neurochir Suppl. 2000;76:81-6.

31. Wong A, Dukic-Stefanovic S, Gasic-Milenkovic J, Schinzel R, Wiesinger $\mathrm{H}$, Riederer $\mathrm{P}$, et al. Anti-inflammatory antioxidants attenuate the expression of inducible nitric oxide synthase mediated by advanced glycation endproducts in murine microglia. Eur $\mathrm{J}$ Neurosci. 2001;14(12):1961-7. DOI: http://dx.doi.org/10.1046/j.0953816x.2001.01820.x

32. Ravi R, Somani SM, Rybak LP. Mechanism of cisplatin ototoxicity: antioxidant system. Pharmacol Toxicol. 1995;76(6):386-94. PMID: 7479581 DOI: http://dx.doi.org/10.1111/j.1600-0773.1995.tb00167.x

33. Fukaya $\mathrm{H}$, Kanno $\mathrm{H}$. Experimental studies of the protective effect of Ginkgo biloba extract (GBE) on cisplatin-induced toxicity in rats. Nihon Jibiinkoka Gakkai Kaiho. 1999;102(7):907-17. PMID: 10459293 DOI: http://dx.doi.org/10.3950/jibiinkoka.102.907 